\title{
DICHOTOMIES AND ASYMPTOTIC BEHAVIOUR FOR LINEAR DIFFERENTIAL SYSTEMS
}

BY

\author{
JAMES S. MULDOWNEY ${ }^{1}$
}

\begin{abstract}
Sufficient conditions that a system of differential equations $x^{\prime}=A(t) x$ have a dichotomy usually require that the matrix $A(t)$ be bounded or at least that some restriction be placed on the rate of growth or decay of solutions. Here three sets of necessary and sufficient conditions for a dichotomy which do not impose such a restriction are given in terms of Liapunov functions. Each of the theorems gives practical criteria for a dichotomy including the extension to unbounded matrices of criteria which depend on a concept of diagonal dominance for $A(t)$. An asymptotic analysis is also given for subspaces of the solution set by means of the associated compound equations.
\end{abstract}

1. Introduction. Let $S$ be the real or complex numbers and let $J=\left(\omega_{-}, \omega_{+}\right)$be a real interval which may be bounded or unbounded. Suppose $A$ is an $n \times n$ matrix of continuous functions from $J$ to $S$ and let $X$ be a fundamental matrix solution for the linear homogeneous differential system

$$
x^{\prime}=A(t) x, \quad t \in J .
$$

We let $|\cdot|$ denote any norm on $S^{n}$ and also the corresponding matrix norm. If $\mu_{1}, \mu_{2}$ are continuous real-valued functions on $J$, the system (1.1) will be said to have a $\left(\mu_{1}, \mu_{2}\right)$-dichotomy if there exist supplementary projections $P_{1}, P_{2}$ on $S^{n}$ such that

$$
\left|X(t) P_{i} X^{-1}(s)\right| \leqslant K_{i} \exp \left(\int_{s}^{t} \mu_{i}\right), \quad \text { if }(-1)^{i}(s-t) \geqslant 0, i=1,2,
$$

where $K_{1}, K_{2}$ are positive constants.

In the case that $\mu_{1}, \mu_{2}$ are constants, the system (1.1) is said to have an exponential dichotomy if $\mu_{1}<0<\mu_{2}$ and an ordinary dichotomy if $\mu_{1}=\mu_{2}=0$. The concept of a dichotomy was first formulated and investigated by Massera and Schäffer [9] and has proved to be an important advance in the qualitative theory of linear differential systems.

The condition (1.2) is readily seen to be equivalent to

$$
\begin{gathered}
\left|X(t) P_{i} \xi\right| \leqslant L_{i} \exp \left(\int_{s}^{t} \mu_{i}\right)\left|X(s) P_{i} \xi\right|, \quad \text { if }(-1)^{i}(s-t) \geqslant 0, i=1,2, \\
\left|X(t) P_{i} X^{-1}(t)\right| \leqslant M_{i}
\end{gathered}
$$

Received by the editors January 20, 1982 and, in revised form, February 1, 1983.

1980 Mathematics Subject Classification. Primary 34A30; Secondary 34C30, 34D05, 34D20.

${ }^{1}$ Research supported by NSERC Grant 7197. 
for all vectors $\xi \in S^{n}$, where $L_{i}, M_{i}$ are positive constants. If the projection $P_{i}$ has rank $k_{i}, i=1,2, k_{1}+k_{2}=n$, then the condition (1.3) states that the solution space of (1.1) has two supplementary subspaces $\mathscr{X}_{1}, \mathscr{X}_{2}$, with dimensions $k_{1}, k_{2}$ of solutions $x$ satisfying the inequalities

$$
\begin{aligned}
& |x(t)| \leqslant L_{1} \exp \left(\int_{s}^{t} \mu_{1}\right)|x(s)|, \quad t \geqslant s, \\
& |x(t)| \leqslant L_{2} \exp \left(\int_{s}^{t} \mu_{2}\right)|x(s)|, \quad s \geqslant t,
\end{aligned}
$$

respectively. The condition (1.4) states that supplementary projections from $S^{n}$ onto the subspaces $\left\{x(t): x \in \mathscr{X}_{i}\right\}, i=1,2$, are bounded uniformly with respect to $t \in J$ or, equivalently, that the angle between the spaces $\left\{x(t): x \in \mathscr{X}_{i}\right\}, i=1,2$, is bounded away from zero for $t \in J$ (cf. [3, Lecture 2; 4, p. 156]). Thus for example, if $\mu_{1} \leqslant 0 \leqslant \mu_{2}, \mathscr{X}_{1}$ is a $k_{1}$-dimensional subspace of solutions with respect to which the zero solution is uniformly stable for $t \rightarrow \omega_{+}$and $\mathscr{X}_{2}$ is a $k_{2}$-dimensional subspace of solutions with respect to which the zero solution is uniformly stable for $t \rightarrow \omega_{-}$.

Several criteria for exponential dichotomy are available. However, sufficient conditions usually require that the matrix $A(t)$ be bounded on $J$ or, more generally, that solutions to (1.1) have bounded growth or decay (cf. [3, Lectures 1, 6, 7]). In this paper three sets of necessary and sufficient conditions for a $\left(\mu_{1}, \mu_{2}\right)$-dichotomy are given without any such growth restriction. A criterion using a generalized growth condition is also given.

Further questions about the spaces $\mathscr{X}_{1}, \mathscr{X}_{2}$ are also considered. For a system satisfying (1.5), it is clear that if $t_{0} \in J$, exp $\left(-\int_{t_{0}}^{t} \mu_{1}\right)|x(t)|$ is bounded as $t \rightarrow \omega_{+}$and bounded away from zero as $t \rightarrow \omega_{-}$for all nontrivial solutions $x \in \mathscr{X}_{1}$. It is of interest to enquire if there are subspaces of $\mathscr{X}_{1}$ of solutions satisfying

$$
\lim _{t \rightarrow \omega,} \exp \left(-\int_{t_{0}}^{t} \mu_{1}\right)|x(t)|=0, \quad \lim _{t \rightarrow \omega} \exp \left(-\int_{t_{0}}^{t} \mu_{1}\right)|x(t)|=\infty
$$

respectively and to discuss the dimensions of the subspaces. Similar questions apply to $\mathscr{X}_{2}$ with the roles of $\omega_{+}, \omega_{-}$interchanged. This type of problem has been addressed in [10] in the case $P_{1}=I, P_{2}=0$ by means of the compound equations associated with (1.1). That approach is extended to the more general situation considered here.

2. Criteria for a dichotomy. We introduce some general definitions and notation before stating the results.

Admissible pairs. A pair of functions $V_{i}(t, x): J \times S^{n} \rightarrow \mathbf{R}, i=1,2$, which are continuous on their domain and locally lipschitzian in $x$ will be said to be admissible if for each $t \in J$ there exist supplementary projections $Q_{1}(t), Q_{2}(t)$ of rank $k_{1}, k_{2}$ independent of $t$ such that

$$
\begin{gathered}
\left|Q_{i}(t)\right| \leqslant N_{i}, \quad i=1,2, \\
\left|Q_{i}(t) x\right|^{r} \leqslant V_{i}(t, x) \leqslant b_{i}\left|Q_{i}(t) x\right|^{r}, \quad i=1,2,
\end{gathered}
$$

for all $(t, x) \in J \times S^{n}$, where $N_{i}, b_{i}, r$ are positive constants. 
When an admissible pair is given, it is clear that the pair $Q_{i}, i=1,2$, and the number $r$ are uniquely determined; we shall always take $N_{i}, b_{i}$ to be the least numbers which satisfy (2.1), (2.2) respectively.

If $V_{1}(t, x), V_{2}(t, x)$ is an admissible pair and $\lambda=\left(\lambda_{1}, \lambda_{2}\right)$, where $\lambda_{i} \geqslant 0$, then we define

$$
V(\lambda ; t, x)=\lambda_{1} V_{1}(t, x)-\lambda_{2} V_{2}(t, x) .
$$

Except when it is necessary to emphasize it, the letter $\lambda$ will be suppressed so that $V(t, x)=V(\lambda ; t, x)$. For any function $U(t, x): J \times S^{n} \rightarrow \mathbf{R}$ we denote

$$
\dot{U}(t, x)=\limsup _{h \rightarrow 0+} \frac{1}{h}[U(t+h, x+h A(t) x)-U(t, x)] .
$$

THEOREM 2.1. If there exists an admissible pair $V_{1}(t, x), V_{2}(t, x)$ and real numbers $l_{1}, l_{2}$ such that $0 \leqslant l_{i} b_{i}<1, i=1,2$, and

$$
\begin{array}{ll}
\dot{V}(\lambda ; t, x) \leqslant \rho_{\lambda}(t) V(\lambda ; t, x), & \text { if } V(\lambda ; t, x) \geqslant 0, \\
\dot{V}(\lambda ; t, x) \leqslant \delta_{\lambda}(t) V(\lambda ; t, x), & \text { if } V(\lambda ; t, x) \leqslant 0
\end{array}
$$

for both $\lambda=\left(1, l_{2}\right)$ and $\lambda=\left(l_{1}, 1\right)$, where $\rho_{\lambda}, \delta_{\lambda}: J \rightarrow \mathbf{R}$ are continuous functions and $\rho_{\lambda}=r \mu_{1}$ if $\lambda=\left(1, l_{2}\right), \delta_{\lambda}=r \mu_{2}$ if $\lambda=\left(l_{1}, 1\right)$, then (1.1) has a $\left(\mu_{1}, \mu_{2}\right)$-dichotomy.

THEOREM 2.2. If there exists a continuous function $\rho: J \rightarrow \mathbf{R}$ with $\mu_{1} \leqslant \rho \leqslant \mu_{2}$, an admissible pair $V_{1}(t, x), V_{2}(t, x)$ and real numbers $l_{1}, l_{2}, 0<l_{i} b_{i}<1, i=1,2$, such that

$$
\begin{array}{ll}
\dot{V}_{1}(t, x) \leqslant \sigma_{\lambda}(t) V_{1}(t, x), & \text { if } \lambda_{1} V_{1}(t, x) \geqslant \lambda_{2} V_{2}(t, x), \\
\dot{V}_{2}(t, x) \geqslant \nu_{\lambda}(t) V_{2}(t, x), & \text { if } \lambda_{1} V_{1}(t, x) \leqslant \lambda_{2} V_{2}(t, x)
\end{array}
$$

for both

$$
\lambda=\left(1, l_{1}\right), \quad \sigma_{\lambda}=r \rho, \quad \nu_{\lambda}=r \mu_{2}
$$

and

$$
\lambda=\left(l_{1}, 1\right), \quad \sigma_{\lambda}=r \mu_{1}, \quad \nu_{\lambda}=r \rho,
$$

then (1.1) has a $\left(\mu_{1}, \mu_{2}\right)$-dichotomy.

THEOREM 2.3. If (1.1) has a $\left(\mu_{1}, \mu_{2}\right)$-dichotomy, then there exists an admissible pair $V_{1}(t, x), V_{2}(t, x)$ such that, for all $(t, x)$

$$
\begin{aligned}
& \dot{V}_{1}(t, x) \leqslant r \mu_{1}(t) V_{1}(t, x), \\
& \dot{V}_{2}(t, x) \geqslant r \mu_{2}(t) V_{2}(t, x) .
\end{aligned}
$$

COROLlaRY 2.4. (a) The conditions given as sufficient for a $\left(\mu_{1}, \mu_{2}\right)$-dichotomy in Theorem 2.1 are also necessary.

(b) When $\mu_{1} \leqslant \mu_{2}$, the conditions given as sufficient for a $\left(\mu_{1}, \mu_{2}\right)$-dichotomy in Theorem 2.2 are also necessary.

(c) The conditions given as necessary for a $\left(\mu_{1}, \mu_{2}\right)$-dichotomy in Theorem 2.3 are also sufficient. 
Proof of Corollary 2.4. This corollary is implied by Theorems $2.1,2.2$ and 2.3. Part (b) is obvious since, if an admissible pair $V_{1}(t, x), V_{2}(t, x)$ satisfies (2.7), then it also satisfies the conditions of Theorem 2.2. Parts (a), (c) follow from the fact that the conditions of Theorem 2.3 imply those of Theorem 2.1 with $l_{1}=l_{2}=0$. The main observation needed to see this is that if $U_{2}(t, x)=-V_{2}(t, x)$, then the second condition of (2.7), $\dot{V}_{2}(t, x) \geqslant r \mu_{2}(t) V_{2}(t, x)$ for all $(t, x)$, implies $\dot{U}_{2}(t, x) \leqslant$ $r \mu_{2}(t) U_{2}(t, x)$ for all $(t, x)$. To prove this observation, consider $v_{2}(t)=V_{2}(t, x(t))$, $u_{2}(t)=U_{2}(t, x(t))=-v_{2}(t)$, where $x(t)$ is any solution of (1.1). From (2.7), $D^{+} v_{2}$ $\geqslant r \mu_{2} v_{2}$ which implies $D_{+} u_{2} \leqslant r \mu_{2} u_{2}$ which in turn implies $D^{+} u_{2} \leqslant r \mu_{2} u_{2}$ since $u_{2}$, $\mu_{2}$ are continuous functions (cf. [13, p. 255]). Thus $\dot{V}_{2}(t, x) \leqslant r \mu_{2}(t) v_{2}(t, x)$ for all $(t, x)$ as asserted since $x(t)$ is an arbitrary solution of (1.1) and $U_{2}(t, \cdot)$ is lipschitzian.

The theorems should be compared with Massera and Schäffer [9, §§92, 93] and Copper [3, Lecture 7]. The most closely related results to this paper are [9, Theorems 92.B, 93.A, 93.B]. The results of $[9, \S 93]$ give necessary and sufficient conditions for an ordinary dichotomy $\left(\mu_{1}=\mu_{2}=0\right)$ which are quite similar to the conditions given in Theorem 2.3 and it is interesting to note that these results do not impose any boundedness or similar restrictions on $A(t)$ either.

The following lemma will be used in the proofs of Theorems 2.1 and 2.2.

Lemma 2.5. Suppose $P_{i}, i=1,2$, and $Q_{i}, i=1,2$, are two pairs of supplementary projections in $S^{n}$ such that

$$
\left|Q_{i}\right| \leqslant N, \quad i=1,2 .
$$

If $\tau<1$ is a number such that

$$
\tau\left|Q_{1} P_{1}\right| \geqslant\left|Q_{2} P_{1}\right|, \quad \tau\left|Q_{2} P_{2}\right| \geqslant\left|Q_{1} P_{2}\right|,
$$

then

$$
\left|P_{i}\right| \leqslant 2 N \frac{1+\tau}{1-\tau}, \quad i=1,2
$$

Proof. From (2.8) and (2.9),

$$
\begin{aligned}
2 N & \geqslant\left|Q_{1}\right|+\left|Q_{2}\right|=\left|Q_{1} P_{1}+Q_{1} P_{2}\right|+\left|Q_{2} P_{1}+Q_{2} P_{2}\right| \\
& \geqslant\left|Q_{1} P_{1}\right|-\left|Q_{1} P_{2}\right|+\left|Q_{2} P_{2}\right|-\left|Q_{2} P_{1}\right| \\
& \geqslant(1-\tau)\left(\left|Q_{1} P_{1}\right|+\left|Q_{2} P_{2}\right|\right) .
\end{aligned}
$$

But

$$
\left|P_{1}\right|=\left|Q_{1} P_{1}+Q_{2} P_{1}\right| \leqslant\left|Q_{1} P_{1}\right|+\left|Q_{2} P_{1}\right| \leqslant(1+\tau)\left|Q_{1} P_{1}\right|,
$$

and similarly $\left|P_{2}\right| \leqslant(1+\tau)\left|Q_{2} P_{2}\right|$, so that

$$
2 N(1+\tau) \geqslant(1-\tau)\left(\left|P_{1}\right|+\left|P_{2}\right|\right),
$$

which implies (2.10).

Proof of Theorem 2.1. Let $t_{0} \in J$ and let

$$
W(\lambda ; x, t)=\exp \left(-\int_{t_{0}}^{t} \rho_{\lambda}\right) V(\lambda ; t, x), \quad \text { if } V(\lambda ; t, x) \geqslant 0
$$




$$
W(\lambda ; t, x)=\exp \left(-\int_{t_{0}}^{t} \delta_{\lambda}\right) V(\lambda ; t, x), \quad \text { if } V(\lambda ; t, x) \leqslant 0 .
$$

It follows from $(2.5)$ that $D^{+} W(t, x(t)) \leqslant 0$ and $W(t, x(t))$ is nonincreasing if $x$ is a solution of (1.1) and $\lambda=\left(1, l_{2}\right)$ or $\lambda=\left(l_{1}, 1\right)$. In particular, if $\tau \in J$ and $0 \neq x(\tau) \in$ $Q_{1}(\tau) S^{n}$, then $Q_{2}(\tau) x(\tau)=0$ and

$$
W(t, x(t)) \geqslant W(\tau, x(\tau))=\lambda_{1} \exp \left(-\int_{t_{0}}^{t} \rho_{\lambda}\right) V_{1}(\tau, x(\tau))>0, \quad \text { if } t \leqslant \tau .
$$

Choose a sequence $\tau_{m} \in J, \tau_{m} \rightarrow \omega_{+}$; it follows that for each $m$ there is a $k_{1}$-dimensional subspace of solutions $x$ to $(1.1)$ for which $W(t, x(t))$ is nonnegative and nonincreasing, $\omega_{-}<t \leqslant \tau_{m}$. Let $Y_{m}$ be an $n \times k_{1}$ solution matrix of (1.1) for which the columns span this subspace and the columns of $Y_{m}\left(\tau_{0}\right)$ are orthonormal. By compactness of the unit sphere in $S^{n}$, a subsequence of $Y_{m}\left(\tau_{0}\right)$ (without loss of generality, the sequence itself) converges to a matrix $Y\left(\tau_{0}\right)$ whose $k_{1}$ columns are orthonormal. Therefore $\lim _{m \rightarrow \infty} Y_{m}(t)=Y(t)$, for each $t \in J$, where $Y$ is an $n \times k_{1}$ solution matrix of (1.1) which has rank $k_{1}$. If $x_{m}(t)=Y_{m}(t) \xi$, and $x(t)=Y(t) \xi$, $\xi \in S^{k_{1}}$, then $W\left(t, x_{m}(t)\right) \geqslant 0, \omega_{-}<t \leqslant \tau_{m}$, implies $W(t, x(t)) \geqslant 0, \omega_{-}<t<\omega_{+}$. This analysis is valid for both $\lambda=\left(1, l_{2}\right)$ and $\lambda=\left(l_{1}, 1\right)$ so that if $x \in \mathscr{X}_{1}=Y S^{k_{1}}$, a $k_{1}$-dimensional subspace of the solution set to $(1.1)$, then

$$
V_{1}(t, x(t))-l_{2} V_{2}(t, x(t)) \geqslant 0
$$

and

$$
l_{1} V_{1}(t, x(t))-V_{2}(t, x(t)) \geqslant 0, \quad \omega_{-}<t<\omega_{+} .
$$

Therefore $W(\lambda ; t, x(t))=\exp \left(-\int_{t_{0}}^{t} \rho_{\lambda}\right) V(\lambda ; t, x(t))$ and this function is nonincreasing on $J$ for both $\lambda=\left(1, l_{2}\right)$ and $\lambda=\left(l_{1}, 1\right)$. In particular, for $\lambda=\left(1, l_{2}\right)$

$$
V_{1}(t, x(t))-l_{2} V_{2}(t, x(t)) \leqslant \exp \left(\int_{s}^{t} r \mu_{1}\right)\left[V_{1}(s, x(s))-l_{2} V_{2}(s, x(s))\right], \quad t \geqslant s,
$$

which, from (2.12), implies

$$
\left(1-l_{1} l_{2}\right) V_{1}(t, x(t)) \leqslant \exp \left(\int_{s}^{t} r \mu_{1}\right) V_{1}(s, x(s)), \quad t \geqslant s .
$$

Note that $b_{i} \geqslant 1$ and therefore $0<l_{i}<1$, so that $1-l_{1} l_{2}>0$ and so, from (2.2)

$$
\left|Q_{1}(t) x(t)\right| \leqslant b_{1}^{1 / r}\left(1-l_{1} l_{2}\right)^{-1 / r} \exp \left(\int_{s}^{t} \mu_{1}\right)\left|Q_{1}(s) x(s)\right|, \quad t \geqslant s .
$$

From (2.2) and (2.12),

$$
\left(l_{1} b_{1}\right)^{1 / r}\left|Q_{1}(t) x(t)\right| \geqslant\left|Q_{2}(t) x(t)\right|, \quad t \in J, x \in \mathscr{X}_{1},
$$

and therefore

$$
\begin{aligned}
|x(t)| & =\left|Q_{1}(t) x(t)+Q_{2}(t) x(t)\right| \\
& \leqslant\left|Q_{1}(t) x(t)\right|+\left|Q_{2}(t) x(t)\right| \\
& \leqslant\left|Q_{1}(t) x(t)\right|\left[1+\left(l_{1} b_{1}\right)^{1 / r}\right] .
\end{aligned}
$$


This, together with $\left|Q_{1}(s) x(s)\right| \leqslant N_{1}|x(s)|$ (from (2.1)) and (2.13), gives

$$
|x(t)| \leqslant L_{1} \exp \left(\int_{s}^{t} \mu_{1}\right)|x(s)|, \quad t \geqslant s, x \in \mathscr{X}_{1},
$$

where $L_{1}=b_{1}^{1 / r}\left(1-l_{1} l_{2}\right)^{-1 / r}\left[1+\left(l_{1} b_{1}\right)^{1 / r}\right] N_{1}$.

A similar argument shows that there is a $k_{2}$-dimensional subspace $\mathscr{X}_{2}$ of the solutions to (1.1) such that, in analogy to (2.14), (2.15)

$$
\begin{gathered}
\left(l_{2} b_{2}\right)^{1 / r}\left|Q_{2}(t) x(t)\right| \geqslant\left|Q_{1}(t) x(t)\right|, \quad t \in J, x \in \mathscr{X}_{2}, \\
|x(t)| \leqslant L_{2} \exp \left(\int_{s}^{t} \mu_{2}\right)|x(s)|, \quad s \geqslant t, x \in \mathscr{X}_{2} .
\end{gathered}
$$

Inequalities (2.14), (2.16) show that the spaces $\mathscr{X}_{1}, \mathscr{X}_{2}$ are supplementary and therefore, from (2.15), (2.17), there exist supplementary projections $P_{1}, P_{2}$ in $S^{n}$ such that (1.3) holds. Finally (2.1), (2.14), (2.16) show that the conditions of Lemma 2.5 are satisfied for each $t \in J$ by the projections $Q_{i}(t), P_{i}(t)=X(t) P_{i} X^{-1}(t)$ with $\tau=\max \left\{\left(l_{1} b_{1}\right)^{1 / r},\left(l_{2} b_{2}\right)^{1 / r}\right\}, N=\max \left\{N_{1}, N_{2}\right\}$. Therefore (2.10) shows that (1.4) is satisfied.

ProOF OF TheOrem 2.2. We tentatively assume that $\rho=0$. Let $x$ be any solution of (1.1). First observe that for any interval $I \subset J, V_{1}(t, x(t))$ is nonincreasing on $I$ if $V_{1}(t, x(t)) \geqslant l_{2} V_{2}(t, x(t))$, for each $t \in I$. Similarly, $V_{2}(t, x(t))$ is nondecreasing on $I$ if $V_{2}(t, x(t)) \geqslant l_{1} V_{1}(t, x(t))$, for each $t \in I$. We assert that if

$$
l_{1} V_{1}(t, x(t))<V_{2}(t, x(t))
$$

holds for $t=\tau \in J$, then it holds for all $t, \tau \leqslant t<\omega_{+}$. To see this, let

$$
s_{0}=\sup \{s:(2.18) \text { holds, } \tau \leqslant t \leqslant s\} .
$$

By continuity, $\tau<s_{0} \leqslant \omega_{+}$and

$$
l_{1} V_{1}\left(s_{0}, x\left(s_{0}\right)\right)=V_{2}\left(s_{0}, x\left(s_{0}\right)\right)
$$

if $s_{0}<\omega_{+}$. From (2.2), (2.18) and (2.20), $x \neq 0$ and $V_{1}\left(s_{0}, x\left(s_{0}\right)\right) \neq 0, V_{2}\left(s_{0}, x\left(s_{0}\right)\right)$ $\neq 0$. Therefore, from $(2.20), V_{1}\left(s_{0}, x\left(s_{0}\right)\right)>l_{2} V_{2}\left(s_{0}, x\left(s_{0}\right)\right)$ and, by continuity,

$$
V_{1}(t, x(t))>l_{2} V_{2}(t, x(t))
$$

for $s_{0}-\delta \leqslant t \leqslant s_{0}$ for some $\delta$ with $0<\delta \leqslant s_{0}-\tau$. Thus $V_{1}(t, x(t))$ is nonincreasing and $V_{2}(t, x(t))$ is nondecreasing, $s_{0}-\delta \leqslant t \leqslant s_{0}$, from (2.18), (2.19), (2.21), implying

$$
l_{1} V_{1}(t, x(t)) \geqslant l_{1} V_{1}\left(s_{0}, x\left(s_{0}\right)\right)=V_{2}\left(s_{0}, x\left(s_{0}\right)\right) \geqslant V_{2}(t, x(t)),
$$

$s_{0}-\delta \leqslant t \leqslant s_{0}$, contradicting (2.19). Thus $s_{0}=\omega_{+}$, as asserted. From this assertion it now follows that if

$$
l_{1} V_{1}(t, x(t)) \geqslant V_{2}(t, x(t))
$$

holds for $t=\tau$, then it also holds for all $t, \omega_{-}<t \leqslant \tau$.

If the assumption $\rho=0$ is dropped, the preceding statement about (2.22) may still be proved in the same way if $V_{i}(t, x)$ is replaced by $\exp \left(-\int_{t_{0}}^{t} r \rho\right) V_{i}(t, x), i=1,2$, throughout the proof. 
As in the proof of Theorem 2.1, it follows by considering a sequence $\tau_{m} \rightarrow \omega_{+}$that there exists a $k_{1}$-dimensional subspace $\mathscr{X}_{1}$ of the solutions to (1.1) such that (2.22) holds for all $t \in J$ and all $x \in \mathscr{X}_{1}$. From (2.2), (2.22) we deduce that (2.14) holds for all $x \in \mathscr{X}_{1}$ and, from (2.2), (2.6), (2.22), that (2.15) holds for all $x \in \mathscr{X}_{1}$, with $L_{1}=b_{1}^{1 / r}\left[1+\left(l_{1} b_{1}\right)^{1 / r}\right] N_{1}$. An analogous argument shows the existence of a $k_{2^{-}}$ dimensional subspace $\mathscr{X}_{2}$ of solutions satisfying (2.16), (2.17) and the proof may be completed as before.

ProOF OF THEOREM 2.3. This proof is developed from a result on uniform asymptotic stability in $\left[14\right.$, p. 92]. Suppose $(1.1)$ has a $\left(\mu_{1}, \mu_{2}\right)$-dichotomy and let

$$
V_{i}(t, x)=\sup \left\{\left|X(\tau) P_{i} X^{-1}(t) x\right| \exp \left(-\int_{t}^{\tau} \mu_{i}\right):(-1)^{i}(t-\tau) \geqslant 0\right\},
$$

for each $(t, x)$ and $i=1,2$, where $X, P_{i}$ are as in (1.2).

First we show that $V_{1}(t, x), V_{2}(t, x)$ are admissible. Let $Q_{i}(t)=X(t) P_{i} X^{-1}(t)$, $i=1,2$; it is readily verified that $Q_{1}(t), Q_{2}(t)$ are supplementary projections which, from (1.4), satisfy (2.1). Choosing $\tau=t$ in (2.23) shows that

$$
\left|Q_{i}(t) x\right|=\left|X(t) P_{i} X^{-1}(t) x\right| \leqslant V_{i}(t, x), \quad i=1,2 .
$$

With $\xi=X^{-1}(t) x$ and $\tau, t$ replacing $t, s$, respectively, (1.3) implies

$$
\begin{aligned}
\left|X(\tau) P_{i} X^{-1}(t) x\right| & \leqslant L_{i} \exp \left(\int_{t}^{\tau} \mu_{i}\right)\left|X(t) P_{i} X^{-1}(t) x\right| \\
& =L_{i} \exp \left(\int_{t}^{\tau} \mu_{i}\right)\left|Q_{i}(t) x\right|, \quad(-1)^{i}(t-\tau) \geqslant 0,
\end{aligned}
$$

and therefore, from (2.23),

$$
V_{i}(t, x) \leqslant L_{i}\left|Q_{i}(t) x\right|, \quad i=1,2 .
$$

Combining (2.24), (2.25) shows that (2.2) $\left(r=1, b_{i}=L_{i}\right)$ holds. Next, since

$$
\left|X(\tau) P_{i} X^{-1}(t) x\right| \leqslant\left|X(\tau) P_{i} X^{-1}(t)(x-y)\right|+\left|X(\tau) P_{i} X^{-1}(t) y\right|,
$$

it follows from (2.23) that for all $x$ and $y$

$$
V_{i}(t, x) \leqslant V_{i}(t, x-y)+V_{i}(t, y) .
$$

Without loss of generality $V_{i}(t, x) \geqslant V_{i}(t, y)$ so that

$$
\begin{aligned}
\left|V_{i}(t, x)-V_{i}(t, y)\right| & =V_{i}(t, x)-V_{i}(t, y) \leqslant V_{i}(t, x-y) \\
& \leqslant L_{i}\left|Q_{i}(t)(x-y)\right| \text { from }(2.25), \\
& \leqslant L_{i} M_{i}|x-y| \text { from }(1.4),
\end{aligned}
$$

and hence $V_{i}(t, x)$ is lipschitzian in $x$. To see that $V_{1}(t, x)$ is continuous, observe that

$$
\begin{aligned}
V_{1}(t, x) & -V_{1}(s, y) \\
= & {\left[\sup _{\tau \geqslant t}\left|X(\tau) P_{1} X^{-1}(t) x\right| \exp \left(-\int_{t}^{\tau} \mu_{1}\right)-\sup _{\tau \geqslant s}\left|X(\tau) P_{1} X^{-1}(t) x\right| \exp \left(-\int_{t}^{\tau} \mu_{1}\right)\right] } \\
& +\left[\sup _{\tau \geqslant s}\left|X(\tau) P_{1} X^{-1}(t) x\right| \exp \left(-\int_{t}^{\tau} \mu_{1}\right)-\sup _{\tau \geqslant s}\left|X(\tau) P_{1} X^{-1}(s) y\right| \exp \left(-\int_{s}^{\tau} \mu_{1}\right)\right] .
\end{aligned}
$$


Since $\left|X(\tau) P_{1} X^{-1}(t) x\right| \exp \left(-\int_{t}^{\tau} \mu_{1}\right)$ is a bounded continuous function of $\tau, \tau \geqslant t$, the first group of terms in (2.26) is small if $t$ is close to $s$. The second group of terms equals

$$
V_{1}\left(s, X(s) X^{-1}(t) x\right) \exp \left(\int_{s}^{t} \mu_{1}\right)-V_{1}(s, y)
$$

which is small if $(t, x)$ is close to $(s, y)$, because $V_{1}(s, \cdot)$ is lipschitzian; it follows that $V_{1}(t, x)$ is continuous. Similarly $V_{2}(t, x)$ is continuous, completing the proof of admissibility.

Finally it must be shown that (2.7) holds. If $x$ is a solution of (1.1), then for $h>0$,

$$
\begin{aligned}
V_{1}(t+h, x(t+h)) & =\sup _{\tau \geqslant t+h}\left|X(\tau) P_{1} X^{-1}(t+h) x(t+h)\right| \exp \left(-\int_{t+h}^{\tau} \mu_{1}\right) \\
& =\sup _{\tau \geqslant t+h}\left|X(\tau) P_{1} X^{-1}(t) x(t)\right| \exp \left(-\int_{t+h}^{\tau} \mu_{1}\right) \\
& \leqslant \sup _{\tau \geqslant t} X(\tau) P_{1} X^{-1}(t) x(t) \mid \exp \left(-\int_{t+h}^{\tau} \mu_{1}\right) \\
& =V_{1}(t, x(t)) \exp \left(\int_{t}^{t+h} \mu_{1}\right) .
\end{aligned}
$$

Thus

$$
\frac{1}{h}\left[V_{1}(t+h, x(t+h))-V_{1}(t, x(t))\right] \leqslant \frac{1}{h}\left[\exp \left(\int_{t}^{t+h} \mu_{1}\right)-1\right] V_{1}(t, x(t))
$$

and $D^{+} V_{1}(t, x(t)) \leqslant \mu_{1}(t) V_{1}(t, x(t))$ which implies $\dot{V}_{1}(t, x) \leqslant \mu_{1}(t) V_{1}(t, x)$ since $V_{1}(t, x)$ is lipschitzian in $x$. A similar argument gives

$$
D_{-} V_{2}(t, x(t)) \geqslant \mu_{2}(t) V_{2}(t, x(t))
$$

which implies $D^{+} V_{2}(t, x(t)) \geqslant \mu_{2}(t) V_{2}(t, x(t))$ since $V_{2}(t, x(t))$ and $\mu_{2}(t)$ are continuous and therefore

$$
\dot{V}_{2}(t, x) \geqslant \mu_{2}(t) V_{2}(t, x),
$$

completing the proof of the necessity of the conditions of Theorem 2.3 for a $\left(\mu_{1}, \mu_{2}\right)$-dichotomy.

Proposition 2.6. Suppose $H_{i}, i=1,2$, are continuously differentiable, $(n \times n)$ symmetric or hermitean matrix valued functions on $J$ (according as $S=\mathbf{R}$ or $S=\mathbf{C}$ ). If there are constants $l_{i}, b_{i}, i=1,2$, such that $0 \leqslant l_{i} b_{i}<1$ and for each $t \in J$ :

(i) $H_{1}(t) H_{2}(t)=0$,

(ii) $H_{1}(t)+H_{2}(t) \geqslant I$,

(iii) $H_{i}(t) \leqslant h_{i} I, i=1,2$,

(iv) $H(\lambda ; t)=\lambda_{1} H_{1}(t)-\lambda_{2} H_{2}(t)$ satisfies

$$
\begin{array}{ll}
H^{\prime}+A^{*} H+H A \leqslant 2 \mu_{1} H, & \text { if } \lambda=\left(1, l_{2}\right), \\
H^{\prime}+A^{*} H+H A \leqslant 2 \mu_{2} H, & \text { if } \lambda=\left(l_{1}, 1\right),
\end{array}
$$

on $J$, then (1.1) has a $\left(\mu_{1}, \mu_{2}\right)$-dichotomy on $J$. 
Proof. This follows from Theorem 2.1. If rank $H_{i}(t)=k_{i}(t)$, then (i) implies nullity $H_{1}(t) \geqslant k_{2}(t)$ so that $k_{1}(t)+k_{2}(t) \leqslant n$ and (ii) implies $k_{1}(t)+k_{2}(t) \geqslant n$. We conclude $k_{1}(t)+k_{2}(t)=n$, which implies $k_{1}, k_{2}$ are constants since these functions are lower semicontinuous on $J$. By (i), $H_{i}(t)$ commutes with $H_{1}(t)+H_{2}(t)$; therefore $Q_{i}(t)=H_{i}(t)\left[H_{1}(t)+H_{2}(t)\right]^{-1}, i=1,2$, are supplementary symmetric (hermitean) projections of rank $k_{i}, i=1,2$, for each $t \in J$. Since $H_{i}^{2}\left(H_{1}+H_{2}\right)=$ $H_{i}^{3}=H_{i}\left(H_{1}+H_{2}\right)^{2}$, from (i), it follows from (ii), (iii) that $H_{i}^{2} \leqslant H_{i}\left(H_{1}+H_{2}\right)^{2} \leqslant$ $b_{i} H_{i}^{2}$ and hence $Q_{i}^{2} \leqslant H_{i} \leqslant b_{i} Q_{i}^{2}$. Thus the functions $V_{i}(t, x)=x^{*} H_{i}(t) x, i=1,2$, satisfy (2.2) with $r=2$. Also (2.1) is satisfied with $N_{i}=b_{i}$, from (ii), (iii). Condition (iv) implies that (2.5) holds so that all the conditions of Theorem 2.1 are satisfied.

In the following two propositions $a_{i}^{j}$ denotes the entry in the matrix $A$ which is in the $i$ th-row and the $j$ th-column.

Proposition 2.7. Suppose there is a continuous real-valued function $\rho$ on $J$ and constants $l_{i}, 0 \leqslant l_{i}<1, i=1,2$, such that for some $m, 0 \leqslant m \leqslant n$,

$$
\begin{array}{r}
\max \left\{l_{1} \operatorname{Re} a_{j}^{j}+l_{1} \sum_{\substack{i=1 \\
i \neq j}}^{m}\left|a_{i}^{j}\right|+\sum_{i=m+1}^{n}\left|a_{i}^{j}\right|: j=1, \ldots, m\right\} \leqslant l_{1} \rho, \\
\min \left\{l_{2} \operatorname{Re} a_{j}^{j}-\sum_{i=1}^{m}\left|a_{i}^{j}\right|-l_{2} \sum_{\substack{i=m+1 \\
i \neq j}}^{n}\left|a_{i}^{j}\right|: j=m+1, \ldots, n\right\} \geqslant l_{2} \rho .
\end{array}
$$

Then the system (1.1) has a $\left(\mu_{1}, \mu_{2}\right)$-dichotomy, where

$$
\begin{array}{r}
\max \left\{\operatorname{Re} a_{j}^{j}+\sum_{\substack{i=1 \\
i \neq j}}^{m}\left|a_{i}^{j}\right|+l_{2} \sum_{i=m+1}^{n}\left|a_{i}^{j}\right|: j=1, \ldots, m\right\}=\mu_{1}, \\
\min \left\{\operatorname{Re} a_{j}^{j}-l_{1} \sum_{i=1}^{m}\left|a_{i}^{j}\right|-\sum_{\substack{i=m+1 \\
i \neq j}}^{n}\left|a_{i}^{j}\right|: j=m+1, \ldots, n\right\}=\mu_{2} .
\end{array}
$$

Proposition 2.8. Suppose there is a continuous real-valued function $\rho$ on $J$ and constants $l_{i}, 0 \leqslant l_{i}<1, i=1,2$, such that for some $m, 0 \leqslant m \leqslant n$,

$$
\begin{array}{r}
\max \left\{l_{2} \operatorname{Re} a_{i}^{i}+l_{2} \sum_{\substack{j=1 \\
j \neq 1}}^{m}\left|a_{i}^{j}\right|+\sum_{j=m+1}^{n}\left|a_{i}^{j}\right|: i=1, \ldots, m\right\} \leqslant l_{2} \rho, \\
\min \left\{l_{1} \operatorname{Re} a_{i}^{i}-\sum_{j=1}^{m}\left|a_{i}^{j}\right|-l_{1} \sum_{\substack{j=m+1 \\
j \neq i}}^{n}\left|a_{i}^{j}\right|: i=m+1, \ldots, n\right\} \geqslant l_{1} \rho .
\end{array}
$$

Then the system (1.1) has a $\left(\mu_{1}, \mu_{2}\right)$-dichotomy, where

$$
\max \left\{\operatorname{Re} a_{i}^{i}+\sum_{\substack{j=1 \\ j \neq i}}^{m}\left|a_{i}^{j}\right|+l_{1} \sum_{j=m+1}^{n}\left|a_{i}^{j}\right|: i=1, \ldots, m\right\}=\mu_{1},
$$




$$
\min \left\{\operatorname{Re} a_{i}^{i}-l_{2} \sum_{j=1}^{m}\left|a_{i}^{j}\right|-\sum_{\substack{j=m+1 \\ j \neq i}}^{n}\left|a_{i}^{j}\right|: i=m+1, \ldots, n\right\}=\mu_{2} .
$$

Proposition 2.7 follows from Theorem 2.1 with $x=\operatorname{col}\left[x_{1}, \ldots, x_{n}\right], V_{1}(t, x)=\left|x_{1}\right|$ $+\cdots+\left|x_{m}\right|, V_{2}(t, x)=\left|x_{m+1}\right|+\cdots+\left|x_{n}\right|$. Proposition 2.8 follows from Theorem 2.2 with $V_{1}(t, x)=\max \left\{\left|x_{1}\right|, \ldots,\left|x_{m}\right|\right\}, V_{2}(t, x)=\max \left\{\left|x_{m+1}\right|, \ldots,\left|x_{n}\right|\right\}$. These results are motivated by stability theorems for (1.1) which may be deduced by considering as Liapunov functions the norms $\left|x_{1}\right|+\cdots+\left|x_{n}\right|$ and $\max \left\{\left|x_{1}\right|, \ldots,\left|x_{n}\right|\right\}$ (cf. [2, pp. 41, 58, 59]). In verifying these statements, the reader may find it convenient to first check them in the case $\rho=0$ and then transform the general situation to this case by the change of variables $y=\exp \left(-\int_{t_{0}}^{t} \rho\right) x$ in (1.1). It is also worth noting that the two propositions are equivalent in the sense that either proposition can be deduced from the other one by applying the other one to the adjoint system to (1.1), $x^{\prime}=-A^{*}(t) x$, and observing from (1.2) that (1.1) has a $\left(\mu_{1}, \mu_{2}\right)$-dichotomy if and only if the adjoint system has a $\left(-\mu_{2},-\mu_{1}\right)$-dichotomy.

If $x_{1}, \ldots, x_{n}$ are replaced by $T_{1} x, \ldots, T_{N} x$ in the preceding analysis, where $T_{1}, \ldots, T_{N}$ are supplementary projections in $S^{n}$, the following Proposition 2.9 is obtained. For any square matrix $B$, the logarithmic 'norm' of Lozinskii, $\mu(B)$, is defined by

$$
\mu(B)=\lim _{h \rightarrow 0+} \frac{1}{h}[|I+h B|-1] .
$$

Clearly $\mu(B)$ depends on the particular norm $|\cdot|$ used. The most important property of $\mu$ is that, if $x$ is any solution of (1.1) and $v=|x|$, then $-\mu(-A) v \leqslant v_{+}^{\prime} \leqslant \mu(A) v$ on $J$, where $v_{+}^{\prime}$ is the right-hand derivative of $v$. For properties of $\mu$ and some examples, see $[2$, pp. $41,58,59]$.

Proposition 2.9. Suppose that $T_{1}, \ldots, T_{N}$ are supplementary projections in $S^{n}$. Then, subject to any choices of norms in each of the subspaces $T_{i} S^{n}, i=1, \ldots, N$, the results of Propositions 2.7 and 2.8 are still true provided the following notational changes are made in the statements of the propositions:

(i) $N$ replaces $n$ throughout,

(ii) $A_{i}^{j}=T_{i} A T_{j}$ replaces $a_{i}^{j}$,

(iii) $\left|A_{i}^{j}\right|$ is the norm of $A_{i}^{j}$ corresponding to the choices of norms in $T_{i} S^{n}, T_{j} S^{n}$,

(iv) Quantities $\operatorname{Re} a_{i}^{i}$ are replaced by $\mu\left(A_{i}^{i}\right)$ in expressions to be maximized and by $-\mu\left(-A_{i}^{i}\right)$ in expressions which are minimized, where $\mu$ is the Lozinskil 'norm' corresponding to the norm chosen in $T_{i} S^{n}$.

For example, let the system (1.1) be written in the form

$$
u_{1}^{\prime}=A_{1}^{1}(t) u_{1}+A_{1}^{2}(t) u_{2}, \quad u_{2}^{\prime}=A_{2}^{1}(t) u_{1}+A_{2}^{2}(t) u_{2},
$$

where $u_{i}$ are vectors in $S^{k_{i}}$ and $A_{i}^{j}(t)$ are $k_{i} \times k_{j}$ matrices. If, for some $l_{1}, l_{2}$, $0 \leqslant l_{i}<1$, and $\rho$

$$
l_{2} \mu\left(A_{1}^{1}\right)+\left|A_{1}^{2}\right| \leqslant l_{2} \rho, \quad-l_{1} \mu\left(-A_{2}^{2}\right)-\left|A_{2}^{1}\right| \geqslant l_{1} \rho
$$

in (2.27), then the system has a $\left(\mu_{1}, \mu_{2}\right)$-dichotomy, where

$$
\mu\left(A_{1}^{1}\right)+l_{1}\left|A_{1}^{2}\right|=\mu_{1}, \quad-\mu\left(-A_{2}^{2}\right)-l_{2}\left|A_{2}^{1}\right|=\mu_{2} .
$$


This follows from Proposition 2.8 as extended in Proposition 2.9. A similar statement interchanging $A_{1}^{2}$ and $A_{2}^{1}$ in (2.28), (2.29) follows from Proposition 2.7.

EXAMPLE 2.10. Consider the matrix

$$
A(t)=\left[\begin{array}{ccc}
-1 & 0 & 1 / 2 \\
t / 2 & t & t^{2} \\
t / 2 & -t^{2} & t
\end{array}\right], \quad t>0 .
$$

Then (1.1) may be written in the form (2.27) with

$$
A_{1}^{1}=[-1], \quad A_{1}^{2}=[0,1 / 2], \quad A_{2}^{1}=\left[\begin{array}{l}
t / 2 \\
t / 2
\end{array}\right], \quad A_{2}^{2}=\left[\begin{array}{cc}
t & t^{2} \\
-t^{2} & t
\end{array}\right] .
$$

With $V_{1}(t, x)=\left|x_{1}\right|, V_{2}(t, x)=\left|\left(x_{2}, x_{3}\right)\right|=\left(\left|x_{2}\right|^{2}+\left|x_{3}\right|^{2}\right)^{1 / 2}$, we may use Proposition 2.9 in the form (2.28), (2.29). Here $\left|A_{1}^{2}\right|=1 / 2,\left|A_{2}^{1}\right|=t / \sqrt{2}, \mu\left(A_{1}^{1}\right)=-1$ and $-\mu\left(-A_{2}^{2}\right)=t$, the least eigenvalue of the hermitean part of $A_{2}^{2}$ (cf. [2, p. 41]). The conditions (2.28) hold with $l_{1}=1 / \sqrt{2}, l_{2}=1 / 2, \rho=0$ and from (2.29) we conclude that (1.1) has a $(-1+1 / 2 \sqrt{2}, t-t / 2 \sqrt{2})$-dichotomy on $(0, \infty)$.

Dichotomies are preserved under changes of variable in (1.1) of the form $x=$ $\Gamma(t) y$, where $\Gamma$ is a continuously differentiable invertible matrix function which, together with its inverse, is bounded on $J$. Conditions such as those of Proposition 2.9 are not invariant under such transformations and hence further sufficient conditions for a dichotomy may be generated by considering these changes of variable.

3. Growth and decay conditions. While the conditions given in the theorems of the preceding section are both necessary and sufficient for a $\left(\mu_{1}, \mu_{2}\right)$-dichotomy, there may be practical difficulties in finding functions $V_{1}(t, x), V_{2}(t, x)$ satisfying all the conditions of these theorems. Less restrictive conditions are sufficient to imply that (1.3) is satisfied. In the case that $\mu_{1}<0<\mu_{2}$ are constants and solutions to (1.1) have bounded growth or decay (cf. [1, 3, 9]), it is known that (1.3) implies (1.4) and thus (1.3) alone implies an exponential dichotomy. With very little change in the argument, an analogous concept may be defined in the present context to yield some new results even in the case of exponential dichotomies.

Let $\mu$ be a real-valued continuous function on $J$. We will say that solutions to (1.1) have $\mu$-bounded growth [respectively decay] if

$$
\left|X(t) X^{-1}(s)\right| \leqslant C \exp \left(\int_{s}^{t} \mu\right), \quad t \geqslant s[s \geqslant t] .
$$

This is the case with $C=1$, if $\mu=\mu(A)[\mu=-\mu(-A)]$, where $\mu(B)$ is the Lozinskii 'norm' of $B$ corresponding to $|\cdot|$ (cf. [2, p. 58]). The solutions have bounded growth [decay] if (3.1) holds for a constant $\mu$.

Proposition 3.1. Suppose solutions to (1.1) have $\mu$-bounded growth [decay]. Then (1.3) implies (1.4) if, for each $L>1$ there exists $\gamma>0$ such that if $s \in J[t \in J]$ then

$$
\left[L^{-1} \exp \left(\int_{s}^{t} \mu_{2}\right)-L \exp \left(\int_{s}^{t} \mu_{1}\right)\right] \exp \left(-\int_{s}^{t} \mu\right) \geqslant \gamma
$$

for some $t>s[s<t]$. 
Proof. Let solutions to (1.1) have $\mu$-bounded growth and let $\mathscr{X}_{1}, \mathscr{X}_{2}$ be as described in (1.5). If $x^{1} \in \mathscr{X}_{1}, x^{2} \in \mathscr{X}_{2}$ and both solutions are nonzero, then from (1.3) or equivalently (1.5),

$$
\left|\frac{x^{2}(t)}{\left|x^{2}(s)\right|}-\frac{x^{1}(t)}{\left|x^{1}(s)\right|}\right| \geqslant L^{-1} \exp \left(\int_{s}^{t} \mu_{2}\right)-L \exp \left(\int_{s}^{t} \mu_{1}\right), \quad t \geqslant s,
$$

where $L=\max \left\{L_{1}, L_{2}\right\}$ and we have interchanged $s$ and $t$ in the second expression in (1.5). From (3.1)

$$
\left|\frac{x^{2}(t)}{\left|x^{2}(s)\right|}-\frac{x^{1}(t)}{\left|x^{1}(s)\right|}\right| \leqslant\left|\frac{x^{2}(s)}{\left|x^{2}(s)\right|}-\frac{x^{1}(s)}{\left|x^{1}(s)\right|}\right| C \exp \left(\int_{s}^{t} \mu\right), \quad t \geqslant s,
$$

since solutions have $\mu$-bounded growth, and so, if $s \in J$, there exists $t>s$ such that $\left|\frac{x^{2}(s)}{\left|x^{2}(s)\right|}-\frac{x^{1}(s)}{\left|x^{1}(s)\right|}\right| \geqslant\left[L^{-1} \exp \left(\int_{s}^{t} \mu_{2}\right)-L \exp \left(\int_{s}^{t} \mu_{1}\right)\right] C^{-1} \exp \left(-\int_{s}^{t} \mu\right) \geqslant C^{-1} \gamma>0$ if (3.2) holds. This is equivalent to (1.4) (cf. [2, p. 27; 4, p. 156]). The proof in the case of $\mu$-bounded decay is similar.

For example, (3.2) is satisfied if $\mu_{1} \leqslant 0 \leqslant \mu_{2}, \mu \leqslant M\left|\mu_{i}\right|$ for some constant $M$ and $\int_{s}^{\omega_{+}} \mu_{i}$ is divergent for $i=1$ or $i=2$. In this case we can find $t(s)>s$ such that one of

$$
\exp \left(\int_{s}^{t(s)} \mu_{1}\right)=\frac{1}{2} L^{-2} \text { or } \exp \left(\int_{s}^{t(s)} \mu_{2}\right)=2 L^{2}
$$

holds and then

$$
L^{-1} \exp \left(\int_{s}^{t(s)} \mu_{2}\right)-L \exp \left(\int_{s}^{t(s)} \mu_{1}\right) \geqslant \delta>0,
$$

where $\delta=\frac{1}{2} L^{-1}$ or $\delta=L$. Now $\exp \left(\int_{s}^{t(s)} \mu\right) \leqslant\left(2 L^{2}\right)^{M}$, so that (3.2) holds for $t=t(s), \gamma=\delta\left(2 L^{2}\right)^{-M}$.

Proposition 3.2 gives some examples of conditions which imply (1.3) and therefore may be used with Proposition 3.1 to establish dichotomies.

PROPOSITION 3.2. Each of the following is sufficient to imply that solutions to (1.1) satisfy (1.3):

(a) The conditions (2.5) of Theorem 2.1 hold for numbers $l_{1}, l_{2}$ satisfying only $0 \leqslant l_{i} \leqslant 1$ and $l_{1} l_{2}<1$.

(b) The conditions (2.6) of Theorem 2.2 hold only for $\lambda_{1}=\lambda_{2}=1$ and $\mu_{1}<\mu_{2}$ on J.

Statement (a) may be seen from the fact that the only essential use of the condition $l_{i} b_{i}<1$ is in proving (1.4). It can be seen that $\mathscr{X}_{1} \cap \mathscr{X}_{2}=\{0\}$ from the fact that, for $x \neq 0, V_{1}(t, x(t))-l_{2} V_{2}(t, x(t))>0$, if $x \in \mathscr{X}_{1}$ and $l_{1} V_{1}(t, x(t))-$ $V_{2}(t, x(t))<0$ if $x \in \mathscr{X}_{2}$. The statement about $\mathscr{X}_{1}$ claims that inequality (2.11) is strict; if this were not so, then, since $V_{1}(t, x(t))=0, V_{2}(t, x(t))=0$ cannot both hold for any $t$ with $x(t) \neq 0$, we would have $l_{1} V_{1}(t, x(t))-V_{2}(t, x(t))<0$ contradicting (2.12). The statement about $\mathscr{X}_{2}$ is proved similarly. 
To prove (b), as in the proof of Theorem 2.2, we may assume that $\mu_{1}<0<\mu_{2}$. We assert that if (2.22) with $l_{1}=1$ holds strictly for $t=\tau$, it also holds for all $t$, $\omega_{-}<t \leqslant \tau$. If $\omega_{-}<s_{0}=\inf \left\{s:(2.22)\right.$ holds, $\left.l_{1}=1, s \leqslant t \leqslant \tau\right\}$, then $V_{1}\left(s_{0}, x\left(s_{0}\right)\right)$ $=V_{2}\left(s_{0}, x\left(s_{0}\right)\right)$ and, from (2.6) with $\lambda_{1}=\lambda_{2}=1$,

$$
D^{+} V_{1}\left(s_{0}, x\left(s_{0}\right)\right)<0<D^{+} V_{2}\left(s_{0}, x\left(s_{0}\right)\right) \text {. }
$$

This implies that there exists $\delta>0$ such that $V_{1}(s, x(s))<V_{1}\left(s_{0}, x\left(s_{0}\right)\right)$ for all $s \in\left(s_{0}, s_{0}+\delta\right)$ and $V_{2}(s, x(s))>V_{2}\left(s_{0}, x\left(s_{0}\right)\right)$ for some $s \in\left(s_{0}, s_{0}+\delta\right)$. Thus $V_{1}(s, x(s))<V_{2}(s, x(s))$ for some $s \in\left(s_{0}, s_{0}+\delta\right)$, contradicting the definition of $s_{0}$. We conclude $s_{0}=\omega_{-}$. As before we deduce, from the assertion about (2.22), the existence of a $k_{1}$-dimensional subspace $\mathscr{X}_{1}$ of the solutions satisfying the first inequality in (1.5) and an analogous argument gives the existence of a $k_{2}$-dimensional subspace $\mathscr{X}_{2}$ satisfying the second inequality in (1.5). It remains to show $\mathscr{X}_{1} \cap \mathscr{X}_{2}=\{0\}$. This follows from the observation that if $\mu_{1}<\rho<\mu_{2}$, then

$$
\operatorname{Max}\left\{V_{1}(t, x(t)), V_{2}(t, x(t))\right\} \exp \left(-\int_{t_{0}}^{t} \rho\right)=V_{1}(t, x(t)) \exp \left(-\int_{t_{0}}^{t} \rho\right)
$$

is decreasing if $0 \neq x \in \mathscr{X}_{1}$ and

$$
\operatorname{Max}\left\{V_{1}(t, x(t)), V_{2}(t, x(t))\right\} \exp \left(-\int_{t_{0}}^{t} \rho\right)=V_{2}(t, x(t)) \exp \left(-\int_{t_{0}}^{t} \rho\right)
$$

is increasing if $0 \neq x \in \mathscr{X}_{2}$.

For concrete functions $V_{1}(t, x), V_{2}(t, x)$ it is sometimes possible to weaken the hypotheses in results such as Proposition 3.2 by consideration of small perturbations of (1.1). This is illustrated by Proposition 3.3. Here we use the notation

$$
\begin{aligned}
& \mu_{1}=\max \left\{\operatorname{Re} a_{i}^{i}+\sum_{j \neq i}\left|a_{i}^{j}\right|: i=1, \ldots, m\right\}, \\
& \mu_{2}=\min \left\{\operatorname{Re} a_{i}^{i}-\sum_{j \neq i}\left|a_{i}^{j}\right|: i=m+1, \ldots, n\right\}
\end{aligned}
$$

and

$$
\begin{aligned}
\mu=\max \left\{\operatorname{Re} a_{i}^{i}+\sum_{j \neq i}\left|a_{i}^{j}\right|: i=1, \ldots, n\right\} \\
\quad\left[\text { respectively } \mu=\min \left\{\operatorname{Re} a_{i}^{i}-\sum_{j \neq i}\left|a_{i}^{j}\right|: i=1, \ldots, n\right\}\right] .
\end{aligned}
$$

Proposition 3.3. Let $\mu_{1}, \mu_{2}$, $\mu$ be given by (3.3), (3.4).

(a) If $\mu_{1} \leqslant \mu_{2}$ with $\mu_{1}(t)<\mu_{2}(t)$ for some $t \in J$ then the solution set of (1.1) has two supplementary subspaces $\mathscr{X}_{1}, \mathscr{X}_{2}$, with dimensions $m, n-m$, respectively, which satisfy $(1.5)\left(L_{1}=L_{2}=1\right)$ and hence (1.3) holds.

(b) If $\mu_{1}, \mu_{2}, \mu$ also satisfy the condition of Proposition 3.2, then (1.1) has a $\left(\mu_{1}, \mu_{2}\right)$-dichotomy on $J$.

Proof. Consider the systems

$$
x^{\prime}=A(t, \varepsilon) x, \quad t \in J, \varepsilon>0,
$$


where $A(t, \varepsilon)$ is obtained by replacing $a_{i}^{i}$ by $a_{i}^{i}-\varepsilon, i=1, \ldots, m$, and $a_{i}^{i}$ by $a_{i}^{i}+\varepsilon$, $i=m+1, \ldots, n$. With $|x|=\max \left\{\left|x_{1}\right|, \ldots,\left|x_{n}\right|\right\}, \quad V_{1}(t, x)=\max \left\{\left|x_{1}\right|, \ldots,\left|x_{m}\right|\right\}$, $V_{2}(t, x)=\max \left\{\left|x_{m+1}\right|, \ldots,\left|x_{n}\right|\right\}$, the conditions of Proposition 3.2(b) are satisfied with $\mu_{1}, \mu_{2}$ replaced by $\mu_{1}-\varepsilon, \mu_{2}+\varepsilon$. Since $\mu_{1}-\varepsilon<\mu_{2}+\varepsilon$ on $J$, if (2.22) with $l_{1}=1$ holds at $t=\tau$ for any solution of (3.5) then it holds for all $t, \omega_{-}<t \leqslant \tau$. The proof of the existence of the space $\mathscr{X}_{1}$ in (1.5) is now completed as before except that instead of considering a sequence of points $\tau$ converging to $\omega_{+}$we use a sequence of points $(\tau, \varepsilon), \tau \in J, \varepsilon>0$, converging to $\left(\omega_{+}, 0\right)$. The treatment for $\mathscr{X}_{2}$ is similar. The constants $L_{1}, L_{2}$ in (1.5) both equal 1 since $|x(t)|=V_{i}(t, x(t))$ if $x \in \mathscr{X}_{i}$. From $\mu_{1}(t)<\mu_{2}(t)$ for some $t \in J$ and (1.5) $\left(L_{1}=L_{2}=1\right)$ we conclude that $\mathscr{X}_{1} \cap \mathscr{X}_{2}=$ $\{0\}$ so that (1.3) is satisfied. Finally, from (3.4), $\mu=\mu(A)[\mu=-\mu(-A)]$, the Lozinskiĭ 'norm' for the norm $|\cdot|$ specified here. Thus we deduce from Proposition 3.1 that, if $\mu_{1}, \mu_{2}, \mu$ satisfy (3.2), there is a $\left(\mu_{1}, \mu_{2}\right)$-dichotomy for (1.1).

Proposition 3.3 may be used sometimes to prove exponential dichotomy even in the case of unbounded matrices.

EXAmple 3.4. Consider

$$
A(t)=\left[\begin{array}{ccc}
-t^{3}-1 & t^{3} / 2 & t^{3} / 2 \\
1 / 2 & -2 & 1 / 2 \\
0 & t^{2} / 2 & t^{2}
\end{array}\right], \quad 1 \leqslant t<\infty
$$

With $m=2$, one finds

$$
\mu(t)=\frac{3}{2} t^{2}, \quad \mu_{1}(t)=-1, \quad \mu_{2}(t)=\frac{1}{2} t^{2},
$$

which satisfy (3.2) and $\mu_{1}<\mu_{2}$. Thus Proposition 3.3 implies that (1.1) has a $\left(-1, \frac{1}{2} t^{2}\right)$-dichotomy on $[1, \infty)$ and hence an exponential dichotomy. Note that the conditions of Proposition 2.8 are not satisfied.

4. Remarks on diagonal dominance. Theorem 2.2 of this paper was motivated by work on diagonal dominance initiated by Lazer [6] and further developed by Berkey [1] and Palmer [11]. It is shown that if $A$ is bounded on $J$ and the diagonal of $A$ is row dominant in the sense that $\mu_{1} \leqslant-\delta, \mu_{2} \geqslant \delta$ for some $\delta>0$ in (3.3), then (1.1) has an exponential dichotomy on $J$. For bounded matrices, this implies the conditions of Proposition 2.8 with $\mu_{1} \leqslant-\delta, \mu_{2} \geqslant \delta$. Similarly, Proposition 2.7 generalizes the corresponding statement about column dominance to matrices which need not be bounded. The original statement on column dominance is deduced in [1] from the row dominance result for the adjoint of (1.1).

For exponential dichotomies it is useful to think of Propositions 2.7 and 2.8 in the following way. Roughly speaking, they replace the original assumption that $A$ is diagonally dominant and bounded by the weaker assumption that for some $l$, $0<l<1, A$ is diagonally dominant even if we multiply its diagonal entries by $l$. It is clear, however, that the conditions of these propositions are stronger than diagonal dominance alone. Lazer [6] proved that if $A$ is row diagonally dominant, then there are supplementary solution subspaces $\mathscr{X}_{1}, \mathscr{X}_{2}$ to (1.1) such that (1.5) holds with $\mu_{1}, \mu_{2}$ replaced by $-\delta, \delta$. This is also a consequence of Proposition 3.2(b) and of Proposition 3.3(a). Berkey [1] raises the question of whether row diagonal dominance alone 
is sufficient to imply an exponential dichotomy. A negative answer to this question is given by the following example.

EXAMPLE 4.1. Consider

$$
A(t)=\left[\begin{array}{ll}
-t^{2}-1-\frac{1}{2} t^{-1} & t^{2}-1+\frac{1}{2} t^{-1} \\
-t^{2}+1+\frac{1}{2} t^{-1} & t^{2}+1-\frac{1}{2} t^{-1}
\end{array}\right], \quad 1 \leqslant t<\infty .
$$

Note that in (3.3) we now have $\mu_{1}=-2, \mu_{2}=2$ so that $A$ is row diagonally dominant. Also $A$ is column diagonally dominant. A fundamental matrix for (1.1) in this case is given by

$$
X(t)=\left[\begin{array}{ll}
\left(1+t^{-1}\right) e^{-t^{2}} & \left(1-t^{-1}\right) e^{t^{2}} \\
\left(1-t^{-1}\right) e^{-t^{2}} & \left(1+t^{-1}\right) e^{t^{2}}
\end{array}\right] .
$$

We observe that the conclusion of Proposition 3.3(a) holds but that there is no exponential dichotomy since

$$
\lim _{t \rightarrow \infty}\left(\frac{x^{1}(t)}{\left|x^{1}(t)\right|}-\frac{x^{2}(t)}{\left|x^{2}(t)\right|}\right)=0
$$

for all nontrivial solutions $x^{1}, x^{2}$ so that (1.4) is not satisfied.

The equivalence of Propositions 2.7 and 2.8 naturally leads to the speculation that other results related to row dominance, such as Proposition 3.3, might also be true in the context of column dominance. While Proposition 3.3(b) remains true when $\mu_{1}$, $\mu_{2}, \mu$ in (3.3), (3.4) are replaced by the corresponding column expressions (as can be seen by considering the adjoint of (1.1)), Proposition 3.3(a) does not remain true in this circumstance. This is shown by the following example.

EXAMPLE 4.2. Consider

$$
4 A(t)=\left[\begin{array}{cc}
-e^{t}-e^{-t} & 2+e^{t}-e^{-t} \\
2-e^{t}+e^{-t} & e^{t}+e^{-t}
\end{array}\right], \quad 0 \leqslant t<\infty .
$$

A fundamental matrix for (1.1) is given by

$$
X(t)=\left[\begin{array}{ll}
1+e^{-t} & e^{t}-1 \\
1-e^{-t} & e^{t}+1
\end{array}\right] .
$$

The column analogs of the expressions in (3.3) are

$$
\mu_{1}(t)=\frac{1}{2}\left(-1-e^{-t}\right), \quad \mu_{2}(t)=\frac{1}{2}\left(-1+e^{-t}\right)
$$

so that $\mu_{1}<\mu_{2}$. However, the conclusion of a column analog of Proposition 3.3(a) does not hold as can be seen from the fact that no nontrivial solution satisfies $\lim _{t \rightarrow \infty} x(t)=0$ as would be implied if (1.5) held for these $\mu_{1}, \mu_{2}$.

Observe that the actual row expressions in (3.3) for $A(t)$ in Example 4.2 are

$$
\mu_{1}(t)=\frac{1}{2}\left(1-e^{-t}\right), \quad \mu_{2}(t)=\frac{1}{2}\left(1+e^{-t}\right)
$$

and the hypothesis and conclusion of Proposition 3.3(a) are satisfied. However, the resulting exponents in (1.5) are not very sharp.

We note that all results such as Proposition 3.3 related to diagonal dominance can be extended in the spirit of Proposition 2.9 to a concept of block-diagonal dominance. 
5. Compound equations and asymptotic behaviour. In this section we investigate the asymptotic behaviour of certain subspaces of the solution set to (1.1). If $\omega$ is an endpoint of $J$, we define the Conditions $\operatorname{I}(\omega)$ and $\operatorname{II}(\omega)$ for a finite-dimensional linear space $\mathscr{Y}$ of functions on $J$ to $S^{n}$.

Condition $\mathrm{I}(\omega)$. If $y \in \mathscr{Y}$, then:

(i) $\lim \sup _{t \rightarrow \omega}|y(t)|<\infty$,

(ii) $\liminf _{t \rightarrow \omega}|y(t)|=0$ implies $\lim _{t \rightarrow \omega}|y(t)|=0$.

Condition $\operatorname{II}(\omega)$. If $y \in \mathscr{Y}$, then

$$
\limsup _{t \rightarrow \omega}|y(t)|=\infty \text { implies } \lim _{t \rightarrow \omega}|y(t)|=\infty .
$$

If $\mathscr{Y}$ is the solution set to (1.1), then Condition I $(\omega)$ implies Liapunov stability and is implied by uniform stability. More generally, if the solution set to (1.1) has subspaces $\mathscr{X}_{i}, i=1,2$, which satisfy (1.5) and $\mathscr{Y}_{i}, i=1,2$, are the spaces of functions $y(t)=\exp \left(-\int_{t_{0}}^{t} \mu_{i}\right) x(t)$, where $x \in \mathscr{X}_{i}, i=1,2$, respectively, then $\mathscr{Y}_{1}$ has Condition $\mathrm{I}\left(\omega_{+}\right) \wedge \mathrm{II}\left(\omega_{-}\right)$and $\mathscr{Y}_{2}$ has Condition $\mathrm{I}\left(\omega_{-}\right) \wedge \mathrm{II}\left(\omega_{+}\right)$. Moreover, if $\int_{s}^{t} \mu_{1} \leqslant M_{1}$ (a constant) for all $s, t \in J, s \leqslant t$, then $\mathscr{X}_{1}$ has Condition $\mathrm{I}\left(\omega_{+}\right) \wedge \mathrm{II}\left(\omega_{-}\right)$. Similarly, if $\int_{s}^{t} \mu_{2} \geqslant M_{2}$ for all $s, t \in J, s \leqslant t$, then $\mathscr{X}_{2}$ has Condition $\mathrm{I}\left(\omega_{-}\right) \wedge \operatorname{II}\left(\omega_{+}\right)$.

If $Y$ is any $n \times m$ matrix and $1 \leqslant k \leqslant \min \{n, m\}$, then the $k$ th compound $Y^{(k)}$ of $Y$ is the $\left(\begin{array}{l}n \\ k\end{array}\right) \times\left(\begin{array}{c}m \\ k\end{array}\right)$ matrix of all $k \times k$ minors from $Y$, where the order of the rows and columns of $Y^{(k)}$ is the lexicographic order of the sets of $k$ row indices and $k$ column indices, respectively, which determine the minors in $Y$ (cf. [8, p. 16]). If the space $\mathscr{Y}$ has dimension $m$ and $1 \leqslant k \leqslant \min \{n, m\}$, we define $\mathscr{Y}^{(k)}$, the $k$ th compound of $\mathscr{Y}$, as follows: If $Y$ is an $n \times m$ matrix function whose columns are a basis for $\mathscr{Y}$, then $\mathscr{Y}^{(k)}$ is the space of functions from $J$ to $S^{\alpha}, \alpha=\left(\begin{array}{c}n \\ k\end{array}\right)$, which is spanned by the columns of $Y^{(k)}$. This definition is consistent since, if $Z$ is any other matrix with this property with respect to $\mathscr{Y}$, then $Z(t)=Y(t) C$ for each $t \in J$, where $C$ is a constant nonsingular $m \times m$ matrix. Now the Binet-Cauchy Theorem (cf. [8, p. 17]) implies that $Z^{(k)}(t)=Y^{(k)}(t) C^{(k)}$, and $C^{(k)}$ is nonsingular by a theorem of Sylvester $\left(\right.$ cf. $\left[8\right.$, p. 17]) which states that $\operatorname{det}\left[C^{(k)}\right]=[\operatorname{det} C]^{\beta}$, where $\beta=\left(\begin{array}{c}m-1 \\ k-1\end{array}\right)$. Thus $Y^{(k)}$ and $Z^{(k)}$ have the same column space.

By the same argument as in [10, Lemma 2], it may be shown that if $\mathscr{Y}$ satisfies Condition $\mathrm{I}(\omega)$, then $\mathscr{Y}^{(k)}$ satisfies Condition $\mathrm{I}(\omega)$ also. Consequently we may prove the following theorem in the same way as was done in the case $m=n$ in [10].

THEOREM 5.1. Let $Y$ be an m-dimensional linear space of functions from $J$ to $S^{n}$ and let $\omega=\omega_{-}$or $\omega=\omega_{+}$.

(a) Suppose $\mathscr{Y}$ satisfies Condition $\mathrm{I}(\omega)$. Then $\mathscr{Y}$ has an $(m-k+1)$-dimensional subspace of functions $y$ satisfying $\lim _{t \rightarrow \omega}|y(t)|=0$ if all $z \in \mathscr{Y}^{(k)}$ satisfy $\liminf _{t \rightarrow \omega}|z(t)|=0$ and only if all $z \in \mathscr{Y}^{(k)}$ satisfy $\lim _{t \rightarrow \omega}|z(t)|=0$.

(b) Suppose $\mathscr{Y}$ satisfies Condition $\mathrm{II}(\omega)$. Then $\mathscr{Y}$ has an $(m-k+1)$-dimensional subspace whose nonzero elements y satisfy $\lim _{t \rightarrow \omega}|y(t)|=\infty$ if all nonzero $z \in \mathscr{Y}^{(k)}$ satisfy $\lim _{\sup _{t \rightarrow \omega}}|z(t)|=\infty$.

To place Theorem 5.1 in the context of differential equations observe that if $\mathscr{X}$ is the solution space for (1.1) then $\mathscr{X}^{(k)}$ is the solution space for the system

$$
z^{\prime}=A^{[k]}(t) z
$$


where $A^{[k]}=\left[X^{(k)}\right]^{\prime}\left[X^{(k)}\right]^{-1}$ and $X$ is any fundamental matrix for (1.1). This was first pointed out by Schwarz [12]. A precise description of $A^{[k]}$ in terms of the entries in $A$ may be found in $[\mathbf{1 0}, \mathbf{1 2}]$. The reader will find helpful examples of matrices $A^{[k]}$ in [12] and some Lozinskir 'norms' for these matrices are given in [10]. As we have seen, a $\left(\mu_{1}, \mu_{2}\right)$-dichotomy implies the existence of spaces $\mathscr{Y}$ associated with (1.1) satisfying Conditions $\mathrm{I}(\omega), \mathrm{II}(\omega)$, and we may now use Theorem 5.1 to deduce further asymptotic information about these spaces by examining the corresponding spaces $\mathscr{Y}^{(k)}$ associated with (5.1).

We will be primarily interested in the condition (1.5) alone, so the condition that there be a full dichotomy is more restrictive than is necessary for the application of Theorem 5.1. Rather than attempt a full discussion, we give one fairly concrete result and an example which will indicate a general approach to these problems.

For any $n \times n$ matrix $A=\left[a_{i}^{j}\right]$, let $\mu_{1}, \mu_{2}$ be as defined in (3.3). If $1 \leqslant i \leqslant\left(\begin{array}{c}n \\ k\end{array}\right)$, we will write $(i)=\left(i_{1}, \ldots, i_{k}\right)$ to denote the $i$ th number in the lexicographic ordering of all $k$-tuples $\left(i_{1}, \ldots, i_{k}\right)$ such that $1 \leqslant i_{1}<i_{2}<\cdots<i_{k} \leqslant n$. We define the functions $\mu_{k 1}, \nu_{k 1}, \mu_{k 2}, \nu_{k 2}$ by

$$
\begin{aligned}
& \mu_{k 1}=\max \left\{\operatorname{Re}\left(a_{i_{1}}^{i_{1}}+\cdots+a_{i_{k}}^{i_{k}}\right)+\sum_{j \notin(i)}\left(\left|a_{i_{1}}^{j}\right|+\cdots+\left|a_{i_{k}}^{j}\right|\right):(i) \subset(1, \ldots, m)\right\}, \\
& \nu_{k 1}=\min \left\{\operatorname{Re}\left(a_{i_{1}}^{i_{1}}+\cdots+a_{i_{k}}^{i_{k}}\right)-\sum_{j \notin(i)}\left(\left|a_{i_{1}}^{j}\right|+\cdots+\left|a_{i_{k}}^{j}\right|\right):(i) \not \subset(1, \ldots, m)\right\},
\end{aligned}
$$

if $i \leqslant k \leqslant m$,

$$
\begin{aligned}
& \mu_{k 2}=\min \left\{\operatorname{Re}\left(a_{i_{1}}^{i_{1}}+\cdots+a_{i_{k}}^{i_{k}}\right)-\sum_{j \notin(i)}\left(\left|a_{i_{1}}^{j}\right|+\cdots+\left|a_{i_{k}}^{j}\right|\right):(i) \subset(m+1, \ldots, n)\right\}, \\
& \nu_{k 2}=\max \left\{\operatorname{Re}\left(a_{i_{1}}^{i_{1}}+\cdots+a_{i_{k}}^{i_{k}}\right)+\sum_{j \notin(i)}\left(\left|a_{i_{1}}^{j}\right|+\cdots+\left|a_{i_{k}}^{j}\right|\right):(i) \not \subset(m+1, \ldots, n)\right\},
\end{aligned}
$$

if $1 \leqslant k \leqslant n-m$. Here $\sum_{j \notin(i)}$ indicates summation over all indices $j$ which do not occur in $(i)$.

Proposition 5.2. (a) If $\mu_{1} \leqslant \mu_{2}$, then (1.1) has solution subspaces $\mathscr{X}_{1}, \mathscr{X}_{2}$ of dimension $m, n-m$ satisfying (1.5) with $L_{1}=L_{2}=1$. In particular, the sets of functions

$$
\left\{\exp \left(-\int_{t_{0}}^{t} \mu_{1}\right) x(t): x \in \mathscr{X}_{1}\right\}, \quad\left\{\exp \left(-\int_{t_{0}}^{t} \mu_{2}\right) x(t): x \in \mathscr{X}_{2}\right\}
$$

have Condition $\mathrm{I}\left(\omega_{+}\right) \wedge \mathrm{II}\left(\omega_{-}\right)$, Condition $\mathrm{I}\left(\omega_{-}\right) \wedge \mathrm{II}\left(\omega_{+}\right)$respectively.

(b) Suppose $\mu_{1} \leqslant \mu_{2}$ and $\mu_{k 1} \leqslant \nu_{k 1}$. Then the condition

$$
\int_{t_{0}}^{\omega_{+}}\left(\mu_{k 1}-k \mu_{1}\right)=-\infty\left[\int_{\omega_{-}}^{t_{0}}\left(\mu_{k 1}-k \mu_{1}\right)=-\infty\right]
$$


implies

$$
\lim _{t \rightarrow \omega_{+}} \exp \left(-\int_{t_{0}}^{t} \mu_{1}\right)|x(t)|=0 \quad\left[\lim _{t \rightarrow \omega} \exp \left(-\int_{t_{0}}^{t} \mu_{1}\right)|x(t)|=\infty\right]
$$

for all $x[x \neq 0]$ in an $(m-k+1)$-dimensional subspace of $\mathscr{X}_{1}$.

(c) Suppose $\mu_{1} \leqslant \mu_{2}$ and $\mu_{k 2} \geqslant \nu_{k 2}$. Then the condition

$$
\int_{t_{0}}^{\omega_{+}}\left(\mu_{k 2}-k \mu_{2}\right)=\infty \quad\left[\int_{\omega}^{t_{0}}\left(\mu_{k 2}-k \mu_{2}\right)=\infty\right]
$$

implies

$$
\lim _{t \rightarrow \omega_{+}} \exp \left(-\int_{t_{0}}^{t} \mu_{2}\right)|x(t)|=\infty\left[\lim _{t \rightarrow \omega} \exp \left(-\int_{t_{0}}^{t} \mu_{2}\right)|x(t)|=0\right]
$$

for all $x \neq 0[x]$ in an $(n-m-k+1)$-dimensional subspace of $\mathscr{X}_{2}$.

Proof. Proposition 5.2(a) follows essentially from Proposition 3.3(a) except that $\mu_{1}=\mu_{2}$ is not excluded, in which case we cannot conclude that the spaces $\mathscr{X}_{1}, \mathscr{X}_{2}$ are supplementary.

To prove Proposition 5.2(b), we make the assumption that $\mu_{1}=0$. This can always be achieved by the change of variables $y=\exp \left(-\int_{t_{0}}^{t} \mu_{1}\right) x$, which replaces $A(t)$ by $A(t)-\mu_{1}(t) I$ in (1.1) and therefore $\mu_{1}, \mu_{2}$ by $0, \mu_{2}-\mu_{1}$ in (3.3). The corresponding change for (5.1) are that $A^{[k]}(t)$ is replaced by $A^{[k]}(t)-k \mu_{1}(t) I$ and $\mu_{k 1}, \nu_{k 1}$ in (5.2) are replaced by $\mu_{k 1}-k \mu_{1}, \nu_{k 1}-k \mu_{1}$, respectively. Here $I$ represents the identity matrix of the appropriate dimension in each case. We will also consider only the case $\mu_{1}<\mu_{2}$. When $\mu_{1} \leqslant \mu_{2}$, the argument can be modified by considering (3.5) as in the proof of Proposition 3.3.

Now recall how the subspace $\mathscr{X}_{1}$ of the solutions to (1.1) is obtained. Let

$$
\begin{gathered}
|x|=\max \left\{\left|x_{1}\right|, \ldots,\left|x_{n}\right|\right\}, \quad V_{1}(t, x)=\max \left\{\left|x_{1}\right|, \ldots,\left|x_{m}\right|\right\}, \\
V_{2}(t, x)=\max \left\{\left|x_{m+1}\right|, \ldots,\left|x_{n}\right|\right\} .
\end{gathered}
$$

If $x$ is a solution of (1.1) and $\tau \in J$ is such that

$$
|x(\tau)|=V_{1}(\tau, x(\tau))>V_{2}(\tau, x(\tau))=0
$$

then $|x(t)|=V_{1}(t, x(t)) \geqslant V_{2}(t, x(t)), \omega_{-}<t \leqslant \tau$. As in $\S 2$, by considering a sequence $\tau_{l} \rightarrow \omega_{+}$and a sequence of $n \times m$ solution matrices $Y_{l}$ of (1.1) such that $V_{2}\left(\tau_{l}, Y_{l}\left(\tau_{l}\right) \xi\right)=0$, we find an $n \times m$ solution matrix $Y=\lim _{l \rightarrow \infty} Y_{l}$ of $(1.1)$, whose rank is $m$ and such that if $x \in Y S^{m}=\mathscr{X}_{1}$ then $x$ satisfies the first condition of (1.5). Thus $|x|$ is nonincreasing on $J$ (since $\mu_{1}=0$ ) and $\mathscr{X}_{1}$ has Condition $\operatorname{I}\left(\omega_{+}\right) \wedge \operatorname{II}\left(\omega_{-}\right)$. To complete the proof of Proposition 5.2(b) it remains to show that the conditions given there imply

$$
\lim _{t \rightarrow \omega_{+}}|z(t)|=0 \quad\left[\lim _{t \rightarrow \omega}|z(t)|=\infty\right], \quad \text { for all } z \in \mathscr{X}_{1}^{(k)}
$$

$\left[0 \neq z \in \mathscr{X}_{1}^{(k)}\right]$, so that the result follows from Theorem 5.1.

To prove (5.4), let $|z|=\max \left\{\left|z_{1}\right|, \ldots,\left|z_{\alpha}\right|\right\}, U_{1}(t, z)=\max \left\{\left|z_{i}\right|:(i) \subset(1, \ldots, m)\right\}$, $U_{2}(t, z)=\max \left\{\left|z_{i}\right|:(i) \not \subset(1, \ldots, m)\right\}$, if $z \in S^{\alpha}, \alpha=\left(\begin{array}{c}n \\ k\end{array}\right)$. The expressions which are 
maximized in (5.2) to obtain $\mu_{k 1}$ are the row sums for $A^{[k]}$ analogous to the row sums for $A$ which are maximized in (3.3) to obtain $\mu_{1}$ and the rows $i=1, \ldots, m$ of $A$ have been replaced by the rows $(i)$ of $A^{[k]}$ for which $(i) \subset(1, \ldots, m)$. Similarly, $\nu_{k 1}$ is the $A^{[k]}$ analog of the function $\mu_{2}$ for $A$, where the rows $i=m+1, \ldots, n$ in (3.3) are replaced in (5.2) by the rows $(i)$ of $A^{[k]}$ for which $(i) \not \subset(1, \ldots, m)$. Each of the entries in the rows $m+1, \ldots, n$ of the matrix $Y_{l}\left(\tau_{l}\right)$ is zero. Thus any $k \times k$ submatrix of $Y_{l}\left(\tau_{l}\right)$ which contains entries from one or more of these rows has zero determinant. We conclude that the entries in the $(i)$-row of $Y_{l}^{(k)}\left(\tau_{l}\right)$ are all zero if (i) $\not \subset(1, \ldots, m)$. Hence, if $z=Y_{l}^{(k)} \eta, 0 \neq \eta \in S^{\delta}, \delta=\left(\begin{array}{c}m \\ k\end{array}\right)$, then

$$
\left|z\left(\tau_{l}\right)\right|=U_{1}\left(\tau_{l}, z\left(\tau_{l}\right)\right)>U_{2}\left(\tau_{l}, z\left(\tau_{l}\right)\right)=0
$$

and, by an argument analogous to that used to find $\mathscr{X}_{1}$,

$$
|z(t)|=U_{1}(t, z(t)) \geqslant U_{2}(t, z(t))
$$

holds for $\omega_{-}<t \leqslant \tau_{l}$. From this it follows that if $z \in \mathscr{X}_{1}^{(k)}=Y^{(k)} S^{\delta}$, where $Y=$ $\lim _{l \rightarrow \infty} Y_{l}$, then (5.5) holds for all $t \in J$. Therefore, from the definition of $U_{1}, U_{2}$, $\mu_{k 1}$, we have $D^{+}|z(t)| \leqslant \mu_{k 1}(t)|z(t)|, \quad t \in J$, if $z \in \mathscr{X}_{1}^{(k)}$. This implies that $\exp \left(-\int_{t_{0}}^{t} \mu_{k 1}\right)|z(t)|$ is nonincreasing on $J$. Now (5.4) follows from

$$
\int_{t_{0}}^{\omega_{+}} \mu_{k 1}=-\infty \quad\left[\int_{\omega_{-}}^{t_{0}} \mu_{k 1}=-\infty\right],
$$

completing the proof of (b) in the case $\mu_{1}=0$. The statement for general $\mu_{1}$ follows from the change of variables indicated earlier.

The proof of Proposition 5.2(c) is similar.

EXAMPLE 5.3. Let $\mathscr{X}$ be the solution set of (1.1) with

$$
A(t)=\left[\begin{array}{ccc}
-t-1 & t / 2 & t / 2 \\
1 / 2 & -2 & 1 / 2 \\
0 & t^{2} / 2 & t^{2}
\end{array}\right], \quad 1 \leqslant t<\infty .
$$

If $|x|=\max \left\{\left|x_{1}\right|,\left|x_{2}\right|,\left|x_{3}\right|\right\}$, then

$$
\mu(A(t))=\frac{3}{2} t^{2}, \quad \mu(-A(t))=2 t+1,
$$

so that, if $x \in \mathscr{X}, e^{-t^{3} / 2}|x(t)|$ is nonincreasing and $e^{t^{2}+t}|x(t)|$ is nondecreasing. With $m=2, k=2$ in Proposition 5.2 we find

$$
\begin{aligned}
\mu_{1}(t) & =-1, \quad \mu_{2}(t)=\frac{t^{2}}{2} \\
\mu_{k 1}(t) & =-\frac{t}{2}-\frac{5}{2}, \quad \nu_{k 1}(t)=\frac{t^{2}}{2}-\frac{3 t}{2}-1 .
\end{aligned}
$$

Thus $\mathscr{X}$ is the direct sum of two spaces $\mathscr{X}_{1}, \mathscr{X}_{2}$ of dimension 2,1 , respectively, such that $e^{t}|x(t)|$ is nonincreasing if $x \in \mathscr{X}_{1}$ and $e^{-t^{3} / 6}|x(t)|$ is nondecreasing if $x \in \mathscr{X}_{2}$. Further, the space $\mathscr{X}_{1}$ contains at least one nontrivial element $x$ such that $\lim _{t \rightarrow \infty} e^{t}|x(t)|=0$.

The results of $\S 5$ generalize theorems proved for the case $m=n$ in $[2,5,7,10]$.

ACKNOWLEDGEMENT. The author is indebted to the referee for many thoughtful comments which resulted in several improvements in this paper. 


\section{REFERENCES}

1. D. D. Berkey, Comparative exponential dichotomies and column diagonal dominance. J. Math. Anal. Appl. 55 (1976), 140-149.

2. W. A. Coppel, Stability and asymptotic behavior of differential equations, Heath, Boston, Mass., 1965. 3. _ Dichotomies in stability theory, Lecture Notes in Math., vol. 629, Springer-Verlag, New York, 1978.

4. Ju. L. Daleckii and M. G. Krein, Stability of solutions of differential equations in Banach space, Amer. Math. Soc. Transl. 43 (1974).

5. P. Hartman, The existence of large or small solutions of linear differential equations, Duke Math. J. 28 (1961), 421-429.

6. A. C. Lazer, Characteristic exponents and diagonally dominant linear differential systems, J. Math. Anal. Appl. 35 (1971), 215-229.

7. J. W. Macki and J. S. Muldowney, The asymptotic behaviour of solutions to linear s!stems of ordinary differential equations, Pacific J. Math. 33 (1970), 693-706.

8. M. Marcus and H. Minc. A survey of matrix theory and matrix inequalities, Allyn \& Bacon, Boston. Mass., 1964.

9. J. L. Massera and J. J. Schäffer, Linear differential equations and function spaces, Academic Press, New York. 1966.

10. J. S. Muldowney, On the dimension of the zero or infinity tending sets for linear differential equations. Proc. Amer. Math. Soc. 83 (1981), 705-709.

11. K. J. Palmer, A diagonal dominance criterion for exponential dichotomy, Bull. Austral. Math. Soc. 17 (1977), 363-374.

12. B. Schwarz, Totally positive differential systems, Pacific J. Math. 32 (1970), 203-229.

13. E. C. Titchmarsh, The theory of functions, Oxford Univ. Press, London, 1960.

14. T. Yoshizawa, Stability theory by Liapunov's second method, Math. Soc. Japan, Tokyo, 1966.

Department of Mathematics, University of Alberta, Edmonton, Alberta, Canada T6G 2G1 